\title{
Solving of time varying quadratic optimal control problems by using Bézier control points
}

\author{
MORTAZA GACHPAZAN \\ Department of Applied Mathematics, Faculty of Mathematical Sciences, \\ Ferdowsi University of Mashhad, Mashhad, Iran \\ E-mail: gachpazan@ferdowsi.um.ac.ir
}

\begin{abstract}
In this paper, linear quadratic optimal control problems are solved by applying least square method based on Bézier control points. We divide the interval which includes $t$, into $k$ subintervals and approximate the trajectory and control functions by Bézier curves. We have chosen the Bézier curves as piacewise polynomials of degree three, and determined Bézier curves on any subinterval by four control points. By using least square method, we introduce an optimization problem and compute the control points by solving this optimization problem. Numerical experiments are presented to illustrate the proposed method.
\end{abstract}

Mathematical subject classification: $49 \mathrm{~N} 10$.

Key words: Bézier Control Points, Quadratic optimal Control Problem, Least Square.

\section{Introduction}

Optimal control problems arise in a wide variety of disciplines. Apart from traditional areas such as aerospace engineering, robotics and chemical engineering, optimal control theory has also been used with great success in areas as diverse as economics to biomedicine. In particular, finding the analytical solution of optimal control problems are difficult. Thus, numerical methods are needed for the solution of these problems.

The linear quadratic optimal control problems are a class of optimal control and there is an extensive literature on them. There are many papers which their 
authors have given methods for solving linear quadratic optimal control problems. For example, spectral method [10], time-domain decomposition iterative method [16], and etc.

This paper aims at minimizing quadratic cost functionals over solutions of time varying linear systems of the form

$$
\begin{aligned}
& \min I=\frac{1}{2} \boldsymbol{x}^{T}\left(t_{f}\right) H\left(t_{f}\right) \boldsymbol{x}\left(t_{f}\right)+\int_{t_{0}}^{t_{f}}\left(\boldsymbol{x}^{T} P \boldsymbol{x}+\boldsymbol{u}^{T} Q \boldsymbol{u}+K \boldsymbol{x}+R \boldsymbol{u}\right) d t, \\
& \text { s.t. : } \\
& \qquad \begin{aligned}
& \dot{\boldsymbol{x}}=A(t) \boldsymbol{x}+B(t) \boldsymbol{u}+F(t), \\
& \boldsymbol{x}\left(t_{0}\right)=\boldsymbol{x}_{0},
\end{aligned}
\end{aligned}
$$

where $t_{0}$ and $t_{f}$ are given constants and $\boldsymbol{x}(t)=\left(x_{1}(t), \ldots, x_{n}(t)\right)^{T} \in R^{n}, \boldsymbol{u}(t)=$ $\left(u_{1}(t), \ldots, u_{m}(t)\right)^{T} \in R^{m}$ are unknown vectors functions. Also, we assume $H(t)=\left[h_{i j}(t)\right]_{n \times n}, P(t)=\left[p_{i j}(t)\right]_{n \times n}, Q(t)=\left[q_{i j}(t)\right]_{n \times m}, A(t)=\left[a_{i j}(t)\right]_{n \times n}$ and $B(t)=\left[b_{i j}(t)\right]_{n \times m}$ are matrices functions and $K(t)=\left(k_{1}(t), \ldots, k_{n}(t)\right)^{T}$, $R(t)=\left(r_{1}(t), \ldots, r_{m}(t)\right)^{T}$ and $F(t)=\left(f_{1}(t), \ldots, f_{n}(t)\right)^{T}$ are vectors functions, which their all elements are polynomial in $\left[t_{0}, t_{f}\right]$. Also, $x_{0}$ is finite constant vectors and $t_{f}$ is fixed constant. If $\boldsymbol{x}\left(t_{f}\right)=\boldsymbol{x}_{f}$ be given, the first term of the objective function will be converted to a constant and can be omitted.

If $A$ and $B$ independent of $t$, the problem (1) will be called time-invariant [3], and otherwise it called time varying problem, [7], [11], and [21].

Lots of papers and books exist dealing with the Bézier curves or surfaces techniques that are applied to different contexts. Harada [14] and Nürnberger [6] have used the Bézier control points in approximate data and functions. Zheng [13] proposed the use of control points of the Bernstein-Bézier form for solving differential equations numerically and also Evrenosoglu [15] have used this approach for solving singularity perturbed two points boundary value problems. The Bézier curves are used in partial differential equations. For example, the Wave and Heat equations are solved in Bézier form, [1, 2, 12, 18]. Other applications of the Bézier functions and control points are found in $[4,9,19,22]$, that are used in computer aided geometric design and image compression.

We suggest a technique similar to what is in [13] and [15], for solving of linear quadratic optimal control problems. 


\section{Least square method}

We divide the interval $t_{0} \leq t \leq t_{f}$ into a set of grid points such that

$$
t_{j}=t_{0}+j h, \quad j=0,1, \cdots, 2 k,
$$

where $h=\frac{t_{f}-t_{0}}{2 k}$, and $k$ is a positive integer. Let $S_{j}=\left[t_{2 j-2}, t_{2 j}\right]$ for $j=$ $1,2, \cdots, k$, the control problem (1) will be defined piecewise as

$$
\min I_{j}=C_{j}+\int_{t_{2 j-2}}^{t_{2 j}}\left(\boldsymbol{x}_{j}^{T} P \boldsymbol{x}_{j}+\boldsymbol{u}_{j}^{T} Q \boldsymbol{u}_{j}+K \boldsymbol{x}_{j}+R \boldsymbol{u}_{j}\right) d t,
$$

s.t.:

$$
\begin{aligned}
& \dot{\boldsymbol{x}}_{j}=A(t) \boldsymbol{x}_{j}+B(t) \boldsymbol{u}_{j}+F(t), \quad t \in S_{j}, \\
& \boldsymbol{x}_{j}\left(t_{2 j-2}\right)=\mu_{j-1}, \quad \boldsymbol{x}_{j}\left(t_{2 j}\right)=\mu_{j},
\end{aligned}
$$

where $\mu_{0}=\boldsymbol{x}_{0}$ is known, $\mu_{1}, \mu_{2}, \ldots, \mu_{k-1}, \mu_{k}$ are unknown, $\mu_{k}=\boldsymbol{x}_{f}$ and

$$
C_{j}= \begin{cases}\frac{1}{2} \boldsymbol{x}_{k}^{T}\left(t_{f}\right) H\left(t_{f}\right) \boldsymbol{x}_{k}\left(t_{f}\right), & j=k, \\ 0, & j \neq k .\end{cases}
$$

Our strategy is to divide the interval $S_{j}$ into two subintervals and then using a Bézier spline curve to approximate the solutions $\boldsymbol{x}_{j}(t)$ and $\boldsymbol{u}_{j}(t)$ by $\boldsymbol{v}_{j}(t)$ and $\boldsymbol{w}_{j}(t)$, respectively where $\boldsymbol{v}_{j}(t)$ and $\boldsymbol{w}_{j}(t)$ are given below. Individual Bézier curves that are defined over the subintervals are joined together to form the Bézier spline curve. Let the Bézier segment over $\left[t_{2 j-2+\ell}, t_{2 j-1+\ell}\right]$ be

$$
\left[\begin{array}{l}
\boldsymbol{v}_{2 j-1+\ell}(t) \\
\boldsymbol{w}_{2 j-1+\ell}(t)
\end{array}\right]=\sum_{r=0}^{3}\left[\begin{array}{l}
\boldsymbol{a}_{r}^{2 j-1+\ell} \\
\boldsymbol{b}_{r}^{2 j-1+\ell}
\end{array}\right] B_{r}^{3}\left(\frac{t-t_{2 j-2+\ell}}{h}\right), \quad \ell=0,1,
$$

where

$$
B_{r}^{3}\left(\frac{t-t_{2 j-2+\ell}}{h}\right)=\left(\begin{array}{c}
3 \\
r
\end{array}\right) \frac{1}{h^{3}}\left(t_{2 j-1+\ell}-t\right)^{3-r}\left(t-t_{2 j-2+\ell}\right)^{r},
$$

are the Bernstein polynomials of degree 3 over the interval $\left[t_{2 j-2+\ell}, t_{2 j-1+\ell}\right]$ and $\left[\begin{array}{l}\boldsymbol{a}_{r}^{2 j-1+\ell} \\ \boldsymbol{b}_{r}^{2 j-1+\ell}\end{array}\right]$ are vectors of the control points. Now, by substituting (3) into 
differential equation of problem (2), we define the piecewise residual functions $\boldsymbol{E}_{1,2 j-1+\ell}(t)$ and $\boldsymbol{E}_{2,2 j-1+\ell}(t)$ for $t \in\left[t_{2 j-2+\ell}, t_{2 j-1+\ell}\right]$ and $\ell=0,1$, by

$$
\begin{gathered}
\boldsymbol{E}_{1,2 j-1+\ell}(t)=\dot{\boldsymbol{v}}_{2 j-1+\ell}(t)-A(t) \boldsymbol{v}_{2 j-1+\ell}(t)-B(t) \boldsymbol{w}_{2 j-1+\ell}(t)-F(t), \\
\boldsymbol{E}_{2,2 j-1+\ell}(t)=\boldsymbol{v}_{2 j-1+\ell}^{T} P(t) \boldsymbol{v}_{2 j-1+\ell}+\boldsymbol{w}_{2 j-1+\ell}^{T} Q(t) \boldsymbol{w}_{2 j-1+\ell}+R(t) \boldsymbol{w}_{2 j-1+\ell} .
\end{gathered}
$$

The boundary conditions should be applied to the first and last Bézier segments, i.e.,

$$
\boldsymbol{v}_{2 j-1}\left(t_{2 j-2}\right)=\mu_{j-1}, \quad \boldsymbol{v}_{2 j}\left(t_{2 j}\right)=\mu_{j}
$$

Beside the boundary conditions, there are also continuity constraints imposed on each successive pair of Bézier segments. Since the differential equation is of first order, the continuity of the first derivative of $\boldsymbol{x}$ (or $\boldsymbol{v}$ ) is required and this gives

$$
\boldsymbol{v}_{2 j-1}^{(s)}\left(t_{2 j-1}\right)=\boldsymbol{v}_{2 j}^{(s)}\left(t_{2 j-1}\right), \quad s=0,1, \quad j=1,2, \ldots, k .
$$

Thus the control points $\boldsymbol{a}_{r}^{2 j-1+\ell}$ must satisfy

$$
\begin{aligned}
& \boldsymbol{a}_{0}^{2 j-1}=\mu_{j-1}, \\
& \boldsymbol{a}_{3}^{2 j}=\mu_{j}, \\
& \boldsymbol{a}_{0}^{2 j}=\boldsymbol{a}_{3}^{2 j-1}, \\
& \boldsymbol{a}_{1}^{2 j}=2 \boldsymbol{a}_{3}^{2 j-1}-\boldsymbol{a}_{2}^{2 j-1} .
\end{aligned}
$$

If we consider the continuity of $w$, the following constraints will be added to constraints (4),

$$
\begin{aligned}
& \boldsymbol{b}_{0}^{2 j}=\boldsymbol{b}_{3}^{2 j-1}, \\
& \boldsymbol{b}_{1}^{2 j}=2 \boldsymbol{b}_{3}^{2 j-1}-\boldsymbol{b}_{2}^{2 j-1} .
\end{aligned}
$$

Now, for each $j=1,2, \cdots, k$ we define residual function as follows

$$
E_{j}=\left\|C_{j}\right\|^{2}+\sum_{\ell=0}^{1} \int_{t_{2 j-2+\ell}}^{t_{2 j-1+\ell}}\left(M\left\|\boldsymbol{E}_{1,2 j-1+\ell}(t)\right\|^{2}+\boldsymbol{E}_{2,2 j-1+\ell}(t)\right) d t,
$$

where $\|$.$\| is L_{2}$ norm and $M$ is a big enough number. Our goal is to minimize the residual function over $S_{j}$. Taking the derivative of $E_{j}$ with respect to component of the unknown control points

$$
\boldsymbol{a}_{1}^{2 j-1}, \boldsymbol{a}_{2}^{2 j-1}, \boldsymbol{a}_{3}^{2 j-1}, \boldsymbol{a}_{2}^{2 j}, \boldsymbol{b}_{0}^{2 j-1}, \boldsymbol{b}_{1}^{2 j-1}, \boldsymbol{b}_{2}^{2 j-1}, \boldsymbol{b}_{3}^{2 j-1}, \boldsymbol{b}_{2}^{2 j} \text { and } \boldsymbol{b}_{3}^{2 j}
$$


reduces the problem (5) to solve a linear system include $4 n+6 m$ equations and unknowns where $n$ and $m$ are dimensions of $\boldsymbol{x}$ and $\boldsymbol{u}$, respectively. Solving this system, we have the solution in terms of $\mu_{i}, i=1, \ldots, k-1$. From the continuity of the first derivative of the solutions, we have

$$
\boldsymbol{v}_{2 j}^{\prime}\left(t_{2 j}\right)=\boldsymbol{v}_{2 j+1}^{\prime}\left(t_{2 j}\right), \quad j=1, \ldots, k-1,
$$

which gives a linear equation system of $k-1$ equations with $k-1$ unknowns $\mu_{1}, \ldots, \mu_{k-1}$. Substituting the minimum solution back into (4) we derive the approximate solution of the quadratic optimal control problem.

Note 1: For $j=k$, we consider $\boldsymbol{a}_{3}^{2 k}=\mu_{k}$ as unknown in (5), and we compute derivative of $E_{k}$ with respect to component of $\boldsymbol{a}_{3}^{2 k}$.

Note 2: In problem (1), if $\boldsymbol{x}\left(t_{f}\right)$ be known, then we set $C_{k}=0$ and $\mu_{k}=\boldsymbol{x}\left(t_{f}\right)$ will be known.

Note 3: We can use this method for solving a problem of calculus of variation as follows

$$
\min I=\int_{t_{0}}^{t_{f}}\left(\dot{\boldsymbol{x}}^{T} P \dot{\boldsymbol{x}}+\boldsymbol{x}^{T} Q \boldsymbol{x}+K \dot{\boldsymbol{x}}+R \boldsymbol{x}\right) d t,
$$

with the conditions $\boldsymbol{x}\left(t_{0}\right)=\boldsymbol{x}_{0}$ and $\boldsymbol{x}\left(t_{f}\right)=\boldsymbol{x}_{f}$.

\section{Numerical examples}

In this section, numerical experiments are conducted to validate the proposed method. We have solved two following problems. First, an optimal control problem and then a problem in calculus of variation and in each example, we show the graphs of trajectories and optimal control functions.

Example 1. We consider the problem of minimizing the performance index [8]:

$$
I=\frac{1}{2} \int_{0}^{1} u^{2} d t
$$


on trajectories of the systems

$$
\begin{aligned}
& \dot{x}_{1}=x_{2}+u, \\
& 0=-x_{2}+u,
\end{aligned}
$$

which satisfy the boundary values

$$
x_{1}(0)=1, x_{1}\left(\frac{1}{2}\right)=x_{1}(1)=0 .
$$

We solve this problem with $k=1$ and the continuity of $x_{2}$ is omitted. We obtain the solution of the considered problem:

$$
\begin{aligned}
& x_{1}(t)= \begin{cases}8\left(\frac{1}{2}-t\right)^{3}+16.02400\left(\frac{1}{2}-t\right)^{2} t+7.97601\left(\frac{1}{2}-t\right) t^{2}, & 0 \leq t \leq \frac{1}{2}, \\
0, & \frac{1}{2} \leq t \leq 1,\end{cases} \\
& x_{2}(t)=\left\{\begin{array}{rr}
-7.97601\left(\frac{1}{2}-t\right)^{3}-24.07199\left(\frac{1}{2}-t\right)^{2} t & \\
-24.07199\left(\frac{1}{2}-t\right) t^{2}-7.97601 t^{3}, & 0 \leq t \leq \frac{1}{2}, \\
0, & \frac{1}{2} \leq t \leq 1,
\end{array}\right. \\
& u(t)= \begin{cases}-7.976003\left(\frac{1}{2}-t\right)^{3}-24.07198\left(\frac{1}{2}-t\right)^{2} t & \\
-24.07198\left(\frac{1}{2}-t\right) t^{2}-7.97601 t^{3}, & 0 \leq t \leq \frac{1}{2}, \\
0, & \frac{1}{2} \leq t \leq 1,\end{cases}
\end{aligned}
$$

Min $I=0.2500003248$.

The exact trajectories and control functions are obtained by Kurina, [8] as follows:

$$
\begin{gathered}
x_{1}^{*}(t)=\left\{\begin{array}{ll}
-2 t+1, & 0 \leq t \leq \frac{1}{2}, \\
0, & \frac{1}{2} \leq t \leq 1,
\end{array} \quad x_{2}^{*}(t)=u^{*}(t)= \begin{cases}-1, & 0 \leq t \leq \frac{1}{2}, \\
0, & \frac{1}{2} \leq t \leq 1,\end{cases} \right. \\
\operatorname{Min} I=I^{*}=\frac{1}{4} .
\end{gathered}
$$

The graphs of trajectories and control functions which we obtain, are shown in Figures 1-3. 


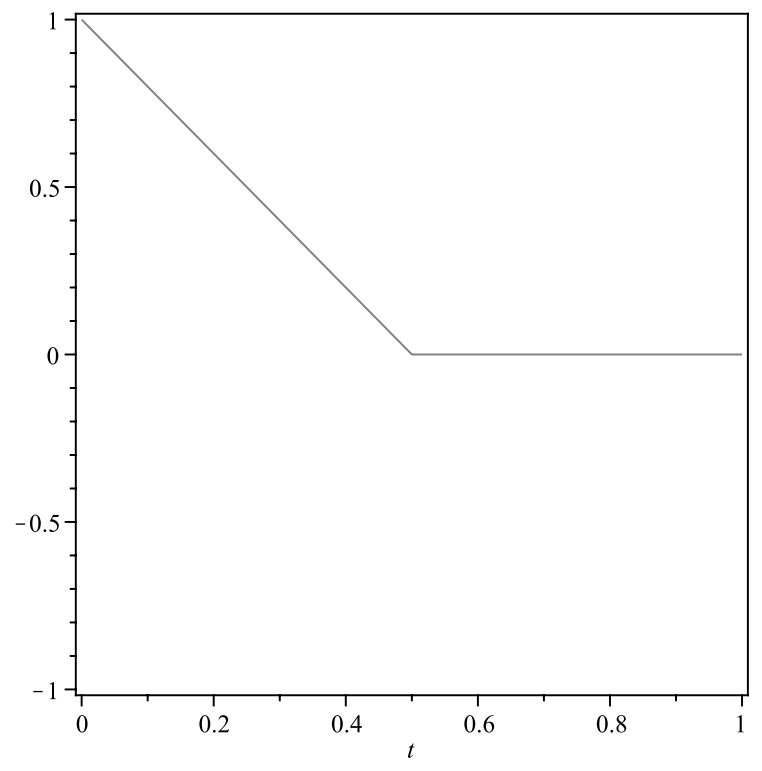

Figure 1 - Graph of trajectory $x_{1}(t)$.

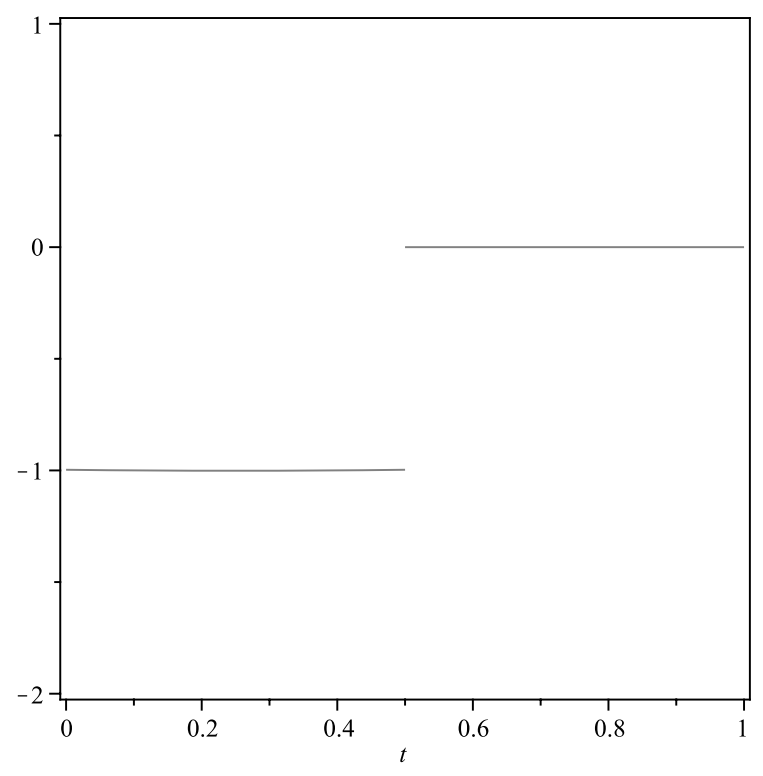

Figure $2-$ Graph of trajectory $x_{2}(t)$. 


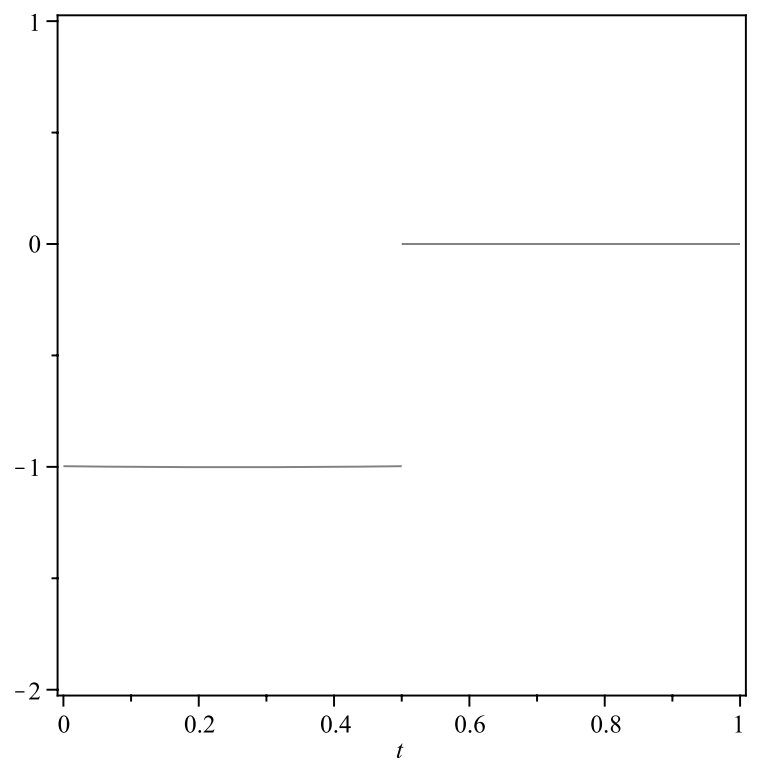

Figure 3 - Graph of control $u(t)$.

Example 2. In second example, we consider a variational problem. We want to find two functions $x_{1}(t)$ and $x_{2}(t)$ which minimize the following objective function, [5]-page 223,

$$
I=\int_{0}^{\frac{\pi}{2}}\left(\dot{x}_{1}^{2}+\dot{x}_{2}^{2}+2 x_{1} x_{2}\right) d t,
$$

subject to:

$$
\begin{array}{ll}
x_{1}(0)=0, & x_{1}\left(\frac{\pi}{2}\right)=1, \\
x_{2}(0)=0, & x_{2}\left(\frac{\pi}{2}\right)=1 .
\end{array}
$$

This problem is solved by $k=1$ and we have obtained $x_{1}(t)=x_{2}(t)$ and Min $I=2.180665422$ for objective function. The approximate solutions of the problem are obtained as:

$$
x_{1}(t)=x_{2}(t)=\left\{\begin{array}{cc}
0.70632 t\left(\frac{\pi}{4}-t\right)^{2}+1.40387 t^{2}\left(\frac{\pi}{4}-t\right) & \\
+0.77943 t^{3}, & 0 \leq t \leq \frac{\pi}{4}, \\
0.77943\left(\frac{\pi}{2}-t\right)^{3}+3.27272\left(\frac{\pi}{2}-t\right)^{2}\left(t-\frac{\pi}{4}\right) & \\
+4.43014\left(\frac{\pi}{2}-t\right)\left(t-\frac{\pi}{4}\right)^{2}+2.06410\left(t-\frac{\pi}{4}\right)^{3}, & \frac{\pi}{4} \leq t \leq \frac{\pi}{2} .
\end{array}\right.
$$


The exact solution of problem and it's value of objective function are:

$$
\begin{gathered}
x_{1}^{*}(t)=x_{2}^{*}(t)=\frac{e^{-\frac{\pi}{2}}\left(e^{t}-e^{-t}\right)}{\left(1-e^{-\pi}\right)}, \quad 0 \leq t \leq \frac{\pi}{2}, \\
\operatorname{Min} I=I^{*}=\frac{2\left(e^{\pi}+1\right)}{e^{\pi}-1}=2.180662822 .
\end{gathered}
$$

The graphs of $x_{1}$ and error function are shown in Figures 4-5.

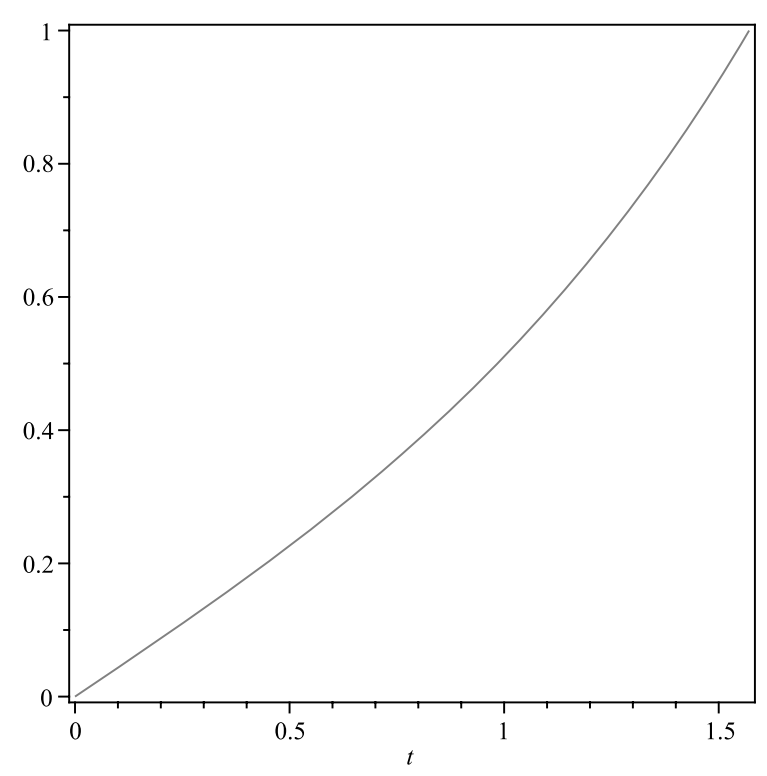

Figure 4-Graph of trajectory $x_{1}(t)=x_{2}(t)$.

Example 3. As a last example, consider a time varying quadratic optimal control problem which is a model of aerospace trajectory control, [20]. The system data is given as follows:

$$
\begin{aligned}
& A(t)=\left[\begin{array}{ccc}
2 t & 1 & \\
0 & & t+1
\end{array}\right], B(t)=\left[\begin{array}{c}
1 \\
\frac{2 t+2}{2 t+3}
\end{array}\right], \\
& P(t)=I, \quad Q(t)=1, x_{0}=(1,1)^{T}, \\
& H(t)=K(t)=R(t)=F(t)=0, \quad t \in[0,4] .
\end{aligned}
$$

We solve this problem by proposed method and obtain the approximate solutions of the problem. For $k=4$, the minimum of the objective function is 


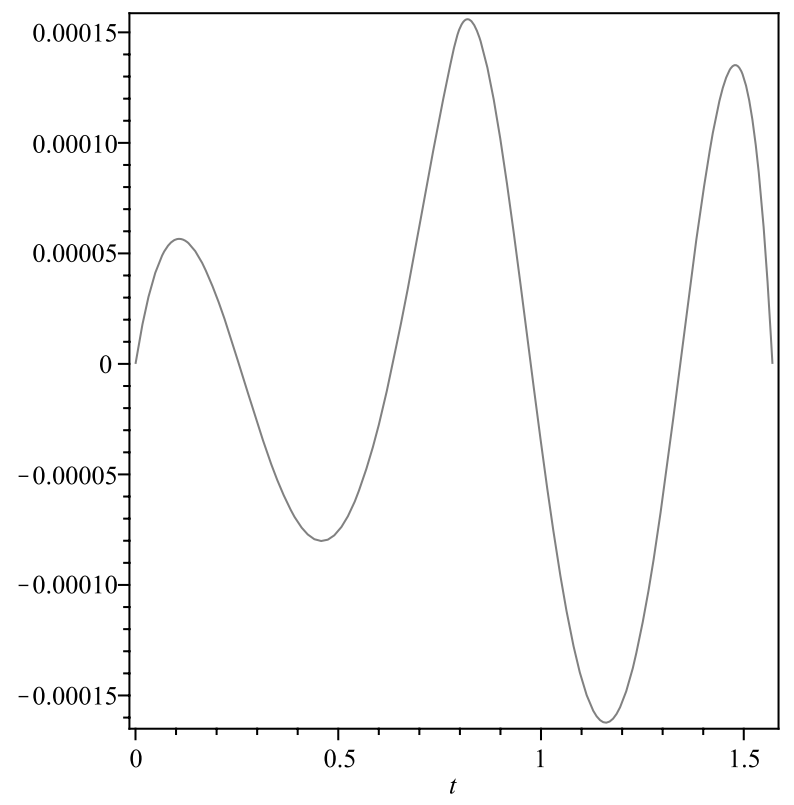

Figure 5 - Graph of error.

6.573994915. Note that, the five terms of Taylor series of $b_{21}$ at $t=2$ is used instead it. The graphs of $x_{1}, x_{2}$ and $u$ are shown in figures 6-8. The asymptotic behaviors of trajectories of the system are discussed in [17] and we see this behaviors in figures.

\section{Conclusions}

We have used least squares methods for numerical solutions of linear quadratic optimal control problems and problems of calculus of variation in quadratic form using Bézier control points instead of computing integrals or performing discretization. The computation is simple and intuitive. The control point structure provides a bound on the residual function. Numerical examples show that the approximate functions are satisfactory for large $h$. Clearly, if we could solve systems of nonlinear equations, this technique will be quite general and can be easily extended to solve other problems, for example, nonlinear quadratic optimal control problems, general problems of calculus of variation. 


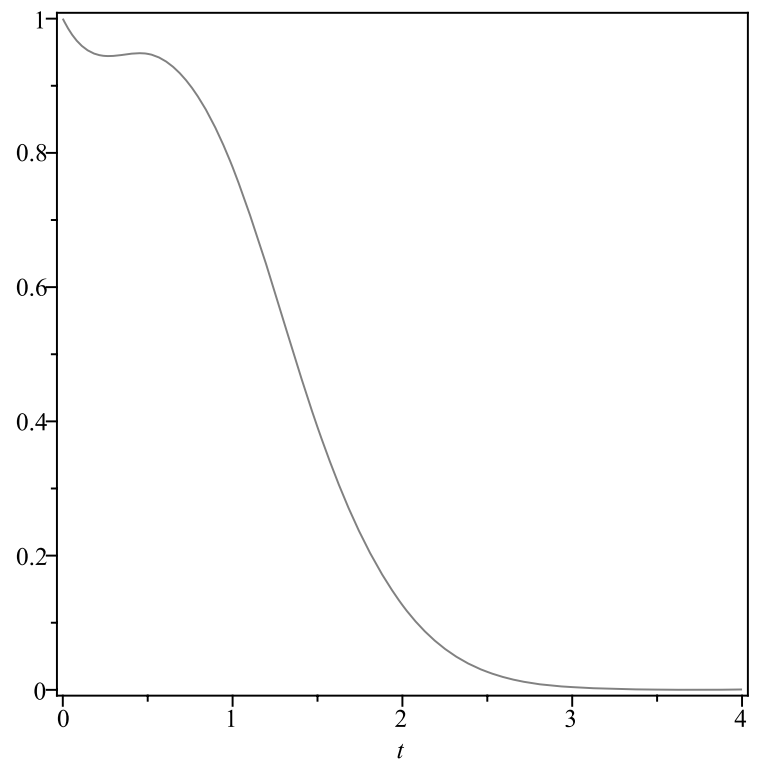

Figure 6 - Graph of trajectory $x_{1}(t)$.

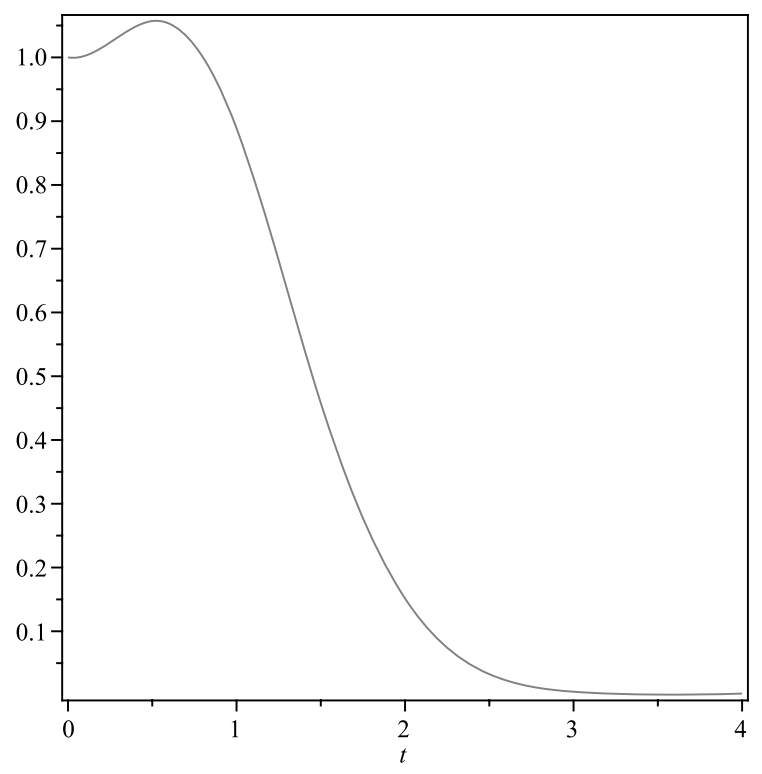

Figure 7 - Graph of trajectory $x_{2}(t)$. 


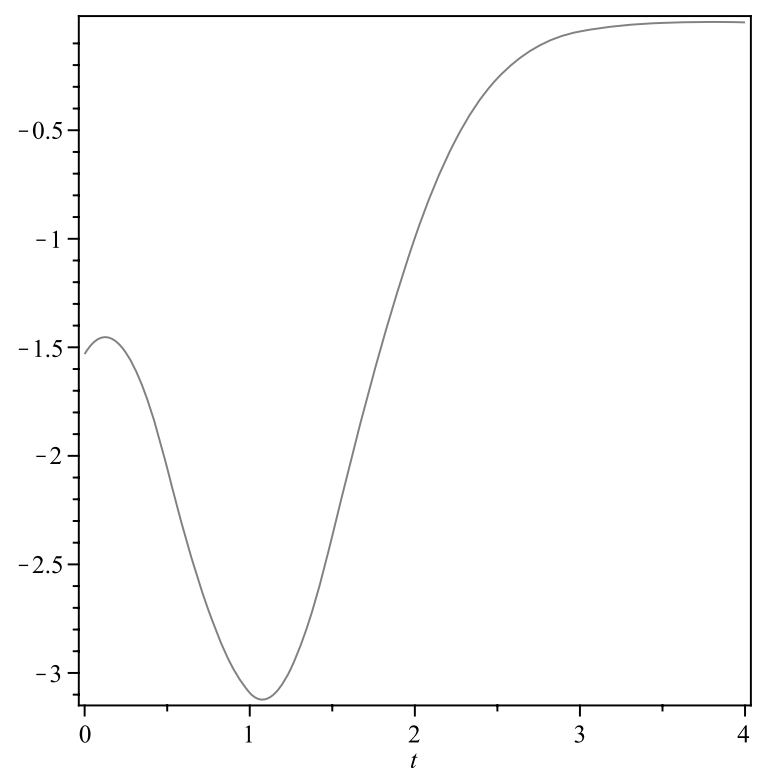

Figure 8 - Graph of control $u(t)$.

\section{REFERENCES}

[1] A.T. Layton and M. van dePanne, A numerically ecient and stable algorithm for animating water waves. The visual Computer, 18 (2002), 41-53.

[2] B. Lang, The synthesis of waveforms using Bézier curves with control point modulation. The Second CEMS Research Student Conference.

[3] B.P. Molinari, The time-invariant linear-quadratic optimal control problems. Automatica, 13 (1977), 347-357.

[4] C.H. Chu, C.C.L. Wang and C.R. Tsai, Computer aided geometric design of strip using developable Bézier patches. Computers in Industry, 59(6) (2008), 601-611.

[5] D. Burghes and A. Graham, Introduction to control theory including optimal control. John Wiley \& Sons (1980).

[6] G. Nürnberger and F. Zeilfelder, Developments in bivariate spline interpolation. Journal of Computational and Applied Mathematics, 121(1-2) (2000), 125-152.

[7] G.A. Kurina and R. Marz, On linear-quadratic optimal control problems for timevarying descriptor systems. SIAM J. Control Optim., 42(6) (2004), 2062-2077.

[8] G.A. Kurina, On some linear-quadratic optimal control problems for descriptor systems, http://www.math.su.se/reports/2006/1. 
[9] G.E. Farin, Curve and surfaces for computer aided geometric design. Academic Press, New York (1988).

[10] H. Juddu, Spectral method for constrained linear-quadratic optimal control. Mathematics and Computers in Simulation, 58 (2002), 159-169.

[11] H.R. Marzban and M. Razzaghi, Hybrid functions approach for linearly constrained quadratic optimal control problems. Applied Mathematical Modelling, 27 (2003), 471-485.

[12] J.V. Beltran and J. Monterde, Bézier solutions of the wave equation. Lecture notes in Computational Sciences, 2(2) (2004), 631-640.

[13] J. Zeng, T.W. Sederberg and R.W. Johnson, Least squares methods for solving differential equations using Bézier control points. Appl. Num. Math., 48 (2004), 237-252.

[14] K. Harada and E. Nakamae, Application of the Bézier curve to data interpolation. Computer-Aided Design, 14(1) (1982), 55-59.

[15] M. Evrenosoglu and S. Somali, Least squares methods for solving singularity perturbed two-points boundary value problems using Bézier control points. Applied Mathematics Letters, 21(10) (2008), 1029-1032.

[16] M. Heinkenschloss, A time-domain decomposition iterative method for the solution of distributed linear quadratic optimal control problems. Journal of Computational and Applied Mathematics, 173 (2005), 169-198.

[17] P. Lu, Closed-form control laws for linear time-varying systems. IEEE Transactions on Automatic Control, 45(3) (2000), 537-542.

[18] R. Cholewa, A.J. Nowak, R.A. Bialecki and L.C. Wrobel, Cubic Bezier splines for BEM heat transfer analysis of the 2-D continuous casting problems. Computational Mechanics, 28 (2002), 282-290.

[19] R. Winkel, Generalized Bernstein Polynomials and Bézier Curves: An Application of Umbral Calculus to Computer Aided Geometric Design. Advances in Applied Mathematics, 27(1) (2001), 51-81.

[20] T. Shu-jun and Z. Wan-xie, Numerical solutions of linear quadratic control for time-varying systems via symplectic conservative perturbation. Applied Mathematics and Mechanics, 28(3) (2007), 277-287.

[21] V. Mehrmann, The autonomous linear quadratic control problems - theory and numerical solution. Lecture Notes in Control and Inform. Sci., 163, SpringerVerlag, Berlin (1991).

[22] Y.Q. Shi and H. Sun, Image and video compression for multimedia engineering. CRC press LLc (2000). 